\title{
Manual of multi-organ procurement and transplantation
}

\author{
ISBN 978-624-605900-2
}

Edited by N Seneviratne, S Edirisinghe, S Udurawana

The Manual of Multi-Organ Procurement and Transplantation certainly fills a long-standing gap in Sri Lankan surgical literature. Compiled by a team of eminent local experts in the field, the editors and authors make an excellent blend of pioneers and the upcoming specialists who are spearheading advances in organ transplantation with the determination to be on par with the rest of the world.

The first chapter brings with it the nostalgia of walking down memory lane, passing the milestones and obstacles of transplant surgery in Sri Lanka, which the pioneers have overcome during the struggle of conquering this new frontier. It holds valuable lessons for the present and future generations of surgeons who pursue the dream of introducing innovative concepts and technology to the existing system.

Reading through this section makes one realize the resistance enforced to the introduction of the mere concept of organ transplantation and establishing it as a legal procedure in our country. It goes on to chronicle the negative attitude towards concepts such as brain death and deceased donor transplantation. These struggles emphasize the sheer power of conviction, dedication and solid team work, enhanced by sharp communication skills and health education, in establishing any new concept in a country with conservative

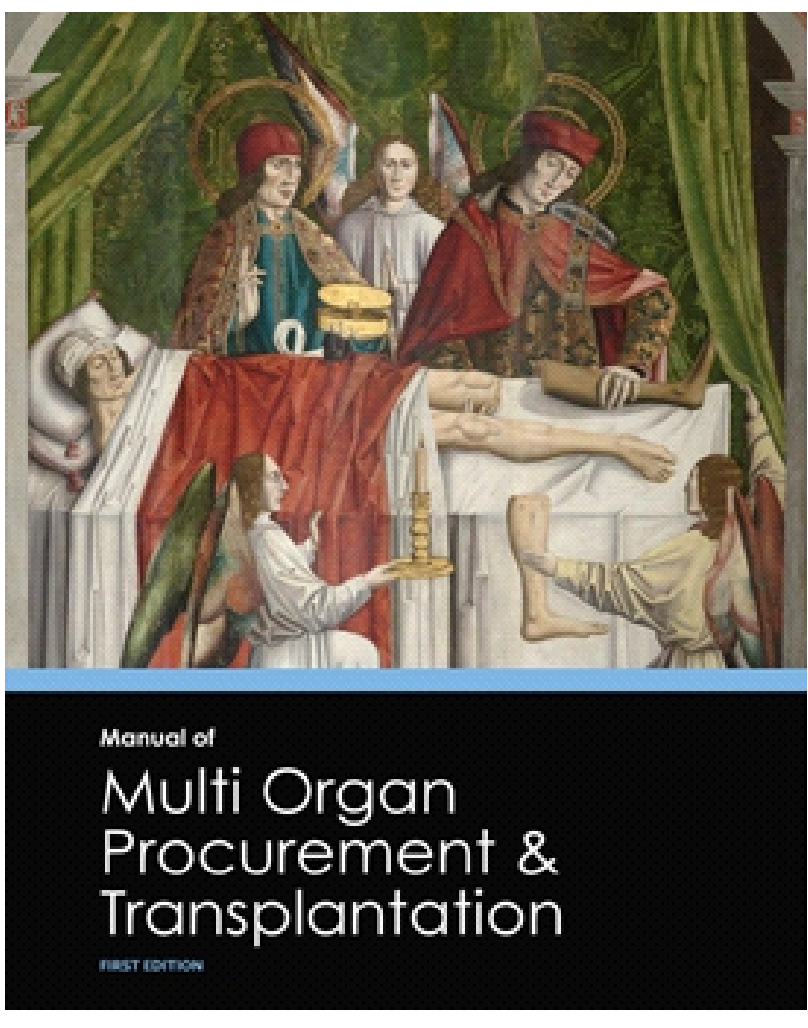
cultural and social norms. This account also elaborates on the expansion of services in the field of organ transplantation starting from the capital and extending to many regional centers driven by the enthusiasm of the younger generations, guided by the senior experts.

The chapter on the structure and process of deceased donor transplantation, written by an expert in medical administration, provides important insights regarding development of the ethical framework and strong legal foundation of the transplant field in Sri Lanka. It also includes useful information regarding the safe and legitimate practice of organ donation.

This book mainly highlights the surgical technical details of commonly performed organ transplants which include kidney, liver, pancreas heart and lungs. Each chapter, which is compiled by local experts specialized in the respective areas, provides the surgical steps of organ retrieval and transplantation in a clear, concise stepwise manner with many tips and tricks that can only come from experienced hands. This format would not only guide a trainee but also immensely assist a specialist consultant at the dawn of his career, providing a quick reference to bolster his confidence before laying the scalpel on the patient.

Chapters on multi-organ retrieval from the deceased donor by itself would provide a valuable hand book for organ retrieval teams not only by emphasizing the essential surgical details but also by way of important information regarding communication and coordination within the transplant team. In the same context, the expertly written chapter on retrieval of abdominal organs draws the main attention to details of commonly performed organ procurements such as the kidney and the liver in Sri Lanka. Details of heart and lung retrieval stimulate the reader to use this knowledge in developing these fields, yet to be firmly established in the developing world. 
It is particularly interesting to read the descriptive, yet succinct, account on living donor transplantation of kidney and liver. Here the experts elaborate each step of organ retrieval, deceased organ explantation (in liver), technique of back bench dissection and ultimately implantation. The elements of upcoming minimal access organ retrieval methods, which would be a promising benchmark procedure for live donors, is also a compelling read.

Details of preoperative workup, post-operative care and especially the management of potential complications were not uniformly presented throughout the chapters, probably due to the necessity of constricting the operative chapters to the steps of performing a perfect operative procedure with minimal complications. As hinted in the preface by the eminent editorship, in future editions one can hope to find a chapter sharing the experience of experts handling these problems. This will be extremely useful for more experienced transplant surgeons.

The chapter on organ preservation highlights the importance of this step in achieving a successful outcome. Principles and techniques of organ cannulation and description of material and solutions provides the basic knowledge needed in this aspect of transplantation. It also adds a note on recent advances in organ preservation solutions, future horizons such as ex-vivo preservation and dynamic continuous perfusion methods which hold much promise in future.

The well written final chapter summarizes almost all the anatomical variations which provides an invaluable guide to all budding transplant surgeons to avoid inadvertent misadventures, especially in multi-organ procurement.

In summary, this well written and skillfully edited manual on organ procurement and transplantation will provide an invaluable practical guide for the surgeons involved in organ transplantation on a full or part time basis in their career. It would also provide a concise reference manual for the post-graduate trainees to be successful in local and overseas examinations.

Reviewed by,

Professor Neville D. Perera, Consultant Urological Surgeon, Institute of Urology and Nephrology, National Hospital of Sri Lanka. nevilledperera@yahoo.com 